\title{
Association of a polymorphism of BTN2A1 with dyslipidemia in community-dwelling individuals
}

\author{
HIDEKI HORIBE ${ }^{1}$, CHIKARA UEYAMA ${ }^{1}$, TETSUO FUJIMAKI ${ }^{2}$, MITSUTOSHI OGURI $^{3}$, \\ KIMIHIKO KATO $^{4,5}$, SAHOKO ICHIHARA ${ }^{5}$ and YOSHIJI YAMADA ${ }^{5}$ \\ ${ }^{1}$ Department of Cardiovascular Medicine, Gifu Prefectural Tajimi Hospital, Tajimi, Gifu 507-8522; \\ ${ }^{2}$ Department of Cardiovascular Medicine, Inabe General Hospital, Inabe, Mie 511-0428; ${ }^{3}$ Department of Cardiology, \\ Japanese Red Cross Nagoya First Hospital, Nagoya, Aichi 453-0046; ${ }^{4}$ Department of Internal Medicine, \\ Meitoh Hospital, Nagoya, Aichi 465-0025; ${ }^{5}$ Department of Human Functional Genomics, \\ Life Science Research Center, Mie University, Tsu, Mie 514-8507, Japan
}

Received May 20, 2013; Accepted January 10, 2014

DOI: $10.3892 / \mathrm{mmr} .2014 .1902$

\begin{abstract}
We have previously shown that the $\mathrm{C} \rightarrow \mathrm{T}$ polymorphism (rs6929846) of the butyrophilin, subfamily 2, member A1 gene (BTN2Al) was significantly associated with myocardial infarction. Considering that dyslipidemia is a significant risk factor for coronary heart disease, it was hypothesized that the association between rs6929846 of $B T N 2 A 1$ and myocardial infarction may be attributable, at least in part, to its effect on the susceptibility to dyslipidemia. The purpose of the present study was to examine a possible association of rs6929846 of BTN2Al with dyslipidemia in community-dwelling individuals. The study subjects were comprised of 5,958 community-dwelling individuals (2,909 subjects with dyslipidemia and 3,049 controls) who were recruited into a population-based cohort study in Inabe, Japan. Dyslipidemia was defined by a serum concentration of triglycerides of $\geq 1.65 \mathrm{mmol} / \mathrm{l}$, a serum high-density lipoprotein-cholesterol concentration of $<1.04 \mathrm{mmol} / \mathrm{l}$ or a serum low-density lipoprotein (LDL)-cholesterol concentration of $\geq 3.64 \mathrm{mmol} / \mathrm{l}$. A comparison of the allele frequencies or genotype distributions by the $\chi^{2}$ test revealed that rs6929846 of BTN2Al was significantly associated with dyslipidemia $(\mathrm{P}<0.05)$. A multivariable logistic regression analysis adjusted for age, gender, body mass index, smoking status and the prevalence of diabetes mellitus revealed that rs6929846 of BTN2Al was significantly (dominant model; $\mathrm{P}=2.4 \times 10^{-4}$; odds ratio, 1.29) associated with dyslipidemia, with the minor $\mathrm{T}$ allele representing a risk for this condition. Among all the individuals, the serum concentrations
\end{abstract}

Correspondence to: Professor Yoshiji Yamada, Department of Human Functional Genomics, Life Science Research Center, Mie University, 1577 Kurima-machiya, Tsu, Mie 514-8507, Japan

E-mail: yamada@gene.mie-u.ac.jp

Key words: dyslipidemia, genetics, polymorphism, BTN2A1, cohort study of total cholesterol, triglycerides and LDL-cholesterol were significantly greater for individuals in the combined $\mathrm{CT}$ and TT genotype groups than for those with the CC genotype. $B T N 2 A 1$ may thus be a susceptibility gene for dyslipidemia in community-dwelling individuals.

\section{Introduction}

The interaction between genetic and environmental factors, the latter including a high-fat and high-calorie diet and physical inactivity, causes the multifactorial disorder, dyslipidemia $(1,2)$. Considering that dyslipidemia is a major risk factor of coronary heart disease $(3,4)$, the personalized prevention of dyslipidemia is important in order to reduce the chance of future cardiovascular events.

Although genome-wide association studies (GWASs) have implicated various loci and genes in the predisposition to dyslipidemia in Caucasians (5-7), the genes that confer susceptibility to this condition in Japanese individuals remain to be definitively identified. We previously showed that the $\mathrm{C} \rightarrow \mathrm{T}$ polymorphism (rs6929846) of the BTN2A1 gene was significantly associated with myocardial infarction in Japanese individuals by a GWAS (8). Considering that dyslipidemia is a significant risk factor for coronary heart disease, it was hypothesized that the association between rs6929846 of BTN2Al and myocardial infarction may be attributable, at least in part, to its effect on the genetic susceptibility to dyslipidemia. In the present study, the correlation of rs6929846 of BTN2Al and dyslipidemia in Japanese individuals was examined.

\section{Materials and methods}

Study population. The study subjects were comprised of 5,958 community-dwelling individuals $(2,909$ subjects with dyslipidemia and 3,049 controls) who were recruited to a population-based cohort study (Inabe Health and Longevity Study) in Inabe, Japan, from 2010 to 2012. The individuals with dyslipidemia either had a serum concentration of triglycerides of $\geq 1.65 \mathrm{mmol} / \mathrm{l}$, a serum high-density lipoprotein (HDL)-cholesterol concentration of $<1.04 \mathrm{mmol} / \mathrm{l}$, a serum 
Table I. Characteristics of the 5,958 study subjects.

\begin{tabular}{lccr}
\hline Characteristic & Dyslipidemia & Controls & P-value \\
\hline No. of subjects & 2909 & 3049 & $<0.0001$ \\
Age, years & $55.5 \pm 11.5$ & $49.8 \pm 13.4$ & $<0.0001$ \\
Gender, \% (male/female) & $59.9 / 40.1$ & $51.1 / 48.9$ & $<0.0001$ \\
Body mass index, $\mathrm{kg} / \mathrm{m}^{2}$ & $23.9 \pm 3.4$ & $22.0 \pm 3.1$ & 0.0003 \\
Current or former smoker, $\%$ & 41.7 & 37.1 & $<0.0001$ \\
Hypertension, $\%$ & 35.7 & 20.8 & $<0.0001$ \\
Diabetes mellitus, $\%$ & 11.8 & 5.0 & $<0.0001$ \\
Serum total cholesterol, mmol/1 & $5.59 \pm 0.87$ & $4.78 \pm 0.60$ & $<0.0001$ \\
Serum triglycerides, mmol/l & $1.67 \pm 1.06$ & $1.79 \pm 0.43$ & $<0.0001$ \\
Serum HDL-cholesterol, mmol/1 & $1.51 \pm 0.41$ & $2.80 \pm 0.52$ & $<0.0001$ \\
Serum LDL-cholesterol, mmol/1 & $3.66 \pm 0.83$ & $5.36 \pm 0.79$ & $<0.0001$ \\
Fasting plasma glucose, mmol/l & $5.75 \pm 1.30$ & $5.57 \pm 0.50$ & $<0.0001$ \\
Blood glycosylated hemoglobin, $\%$ & $5.84 \pm 0.72$ & $63.5 \pm 14.6$ & $<0.0001$ \\
Serum creatinine, $\mu$ mol/l & $67.1 \pm 16.6$ & & \\
\hline
\end{tabular}

Quantitative data are means \pm standard deviation. Hypertension: Systolic blood pressure $\geq 140 \mathrm{mmHg}$, diastolic blood pressure $\geq 90 \mathrm{mmHg}$ or taking antihypertensive medication. Diabetes mellitus: Fasting plasma glucose level of $\geq 6.93 \mathrm{mmol} / \mathrm{l}$, blood glycosylated hemoglobin content of $\geq 6.9 \%$ or taking antidiabetes medication. HDL, high-density lipoprotein; LDL, low-density lipoprotein.

low-density lipoprotein (LDL)-cholesterol concentration of $\geq 3.64 \mathrm{mmol} / 1$ or had been administered antidyslipidemic drugs. The control subjects had normal lipid profiles (a serum triglyceride concentration of $<1.65 \mathrm{mmol} / \mathrm{l}$, a serum HDL-cholesterol concentration of $\geq 1.04 \mathrm{mmol} / 1$ and a serum LDL-cholesterol concentration of $<3.64 \mathrm{mmol} / \mathrm{l}$ ) and no history of dyslipidemia or of taking antidyslipidemic drugs. Diabetes mellitus cases were defined as individuals who either had fasting plasma glucose levels of $\geq 6.93 \mathrm{mmol} / 1$ or a blood glycosylated hemoglobin content of $\geq 6.9 \%$ or as patients who had taken antidiabetes medication. Hypertension cases were defined as individuals who either had a systolic blood pressure of $\geq 140 \mathrm{mmHg}$ or a diastolic blood pressure of $\geq 90 \mathrm{mmHg}$, or as those who had taken antihypertensive medication.

The study protocol complied with the Declaration of Helsinki and was approved by the Committees on the Ethics of Human Research of Mie University Graduate School of Medicine and Inabe General Hospital (Inabe, Japan). Written informed consent was obtained from all subjects.

Measurement of serum lipid profiles. Venous blood was collected in the early morning following overnight fasting by the subjects. The blood samples were centrifuged at $1,600 \mathrm{x}$ for $15 \mathrm{~min}$ at $4^{\circ} \mathrm{C}$, and the serum was separated and stored at $-30^{\circ} \mathrm{C}$ until further analysis. The serum concentrations of total cholesterol, triglycerides, HDL-cholesterol and LDL-cholesterol were measured as previously described (9).

Genotyping of rs6929846 of BTN2A1. Venous blood $(5 \mathrm{ml})$ was collected into tubes containing $50 \mathrm{mmol} / \mathrm{l}$ ethylenediaminetetraacetic acid (disodium salt), then the peripheral blood leukocytes were isolated and the genomic DNA was extracted from these cells with a DNA extraction kit (SMITEST EX-R\&D; Medical \& Biological Laboratories, Nagoya, Japan). The genotypes of rs6929846 of BTN2AI were determined at G\&G Science (Fukushima, Japan) by the multiplex bead-based Luminex assay, a method that combines polymerase chain reaction (PCR) and sequence-specific oligonucleotide probes with suspension array technology (Luminex, Austin, TX, USA). The detailed genotyping methodology has been described previously $(8,10)$.

Statistical analysis. The quantitative data were compared between two groups by unpaired Student's t-test. The categorical data were compared by the $\chi^{2}$ test. The allele frequencies were estimated by the gene counting method, and the $\chi^{2}$ test was used to identify departure from the Hardy-Weinberg equilibrium. Multivariable logistic regression analysis was performed with dyslipidemia, hypertriglycedemia, hypo-HDL-cholesterolemia or hyper-LDL-cholesterolemia as a dependent variable, and with independent variables, including age, gender ( 0 , female; 1 , male), BMI, smoking status $(0$, non-smoker; 1 , current or former smoker) and the prevalence of diabetes mellitus $(0$, no history of this condition; 1 , positive history) and the BTN2Al genotype. The P-value, odds ratio and $95 \%$ confidence interval were calculated. The BTN2A1 genotype was assessed according to dominant, recessive and additive genetic models. Additive models included the additive 1 (heterozygotes versus wild-type homozygotes) and additive 2 (variant homozygotes versus wild-type homozygotes) models, which were analyzed simultaneously with a single statistical model. $\mathrm{P}<0.05$ was considered to indicate a statistically significant difference. Statistical tests were performed with JMP 5.1 software (SAS Institute, Cary, NC, USA).

\section{Results}

Patient characteristics. The characteristics of the study subjects are shown in Table I. The age, frequency of males, 
Table II. Correlation of rs6929846 of BTN2Al to dyslipidemia, hypertriglycedemia, hypo-HDL-cholesterolemia or hyper-LDL-cholesterolemia, as determined by the $\chi^{2}$ test.

\begin{tabular}{|c|c|c|c|c|c|}
\hline \multirow[b]{2}{*}{ Clinical phenotype } & \multicolumn{3}{|c|}{ BTN2A1 genotype, n (\%) } & \multirow{2}{*}{$\begin{array}{l}\text { P-value } \\
\text { (genotype) }\end{array}$} & \multirow{2}{*}{$\begin{array}{c}\text { P-value } \\
\text { (allele frequency) }\end{array}$} \\
\hline & $\mathrm{CC}$ & $\mathrm{CT}$ & TT & & \\
\hline Dyslipidemia & 2265 (77.9) & $599(20.6)$ & $45(1.5)$ & 0.0020 & 0.0004 \\
\hline Controls & $2479(81.3)$ & $540(17.7)$ & $30(1.0)$ & & \\
\hline Hypertriglycedemia & $861(76.5)$ & $253(22.5)$ & $12(1.1)$ & 0.0058 & 0.0119 \\
\hline No hypertriglycedemia & $3883(80.4)$ & $886(18.3)$ & $63(1.3)$ & & \\
\hline Hypo-HDL-cholesterolemia & $236(77.9)$ & $65(21.5)$ & $2(0.7)$ & 0.3790 & 0.6439 \\
\hline No hypo-HDL-cholesterolemia & 4508 (79.7) & $1074(19.0)$ & $73(1.3)$ & & \\
\hline Hyper-LDL-cholesterolemia & $1365(77.9)$ & $360(20.5)$ & $27(1.5)$ & 0.0764 & 0.0236 \\
\hline No hyper-LDL-cholesterolemia & $3379(80.3)$ & $779(18.5)$ & $48(1.1)$ & & \\
\hline
\end{tabular}

Bold denotes values $\mathrm{P}<0.05$. Hypertriglycedemia: A serum triglyceride concentration of $\geq 1.65 \mathrm{mmol} / \mathrm{l}$. Hypo-HDL-cholesterolemia: A serum HDL-cholesterol concentration of $<1.04 \mathrm{mmol} / 1$. Hyper-LDL-cholesterolemia: A serum LDL-cholesterol concentration of $\geq 3.64 \mathrm{mmol} / 1$. BTN2A1, butyrophilin, subfamily 2, member A1 gene; HDL, high-density lipoprotein; LDL, low-density lipoprotein.

Table III. Multivariable logistic regression analysis of rs6929846 of BTN2Al and dyslipidemia, hypertriglycedemia, hypo-HDL-cholesterolemia or hyper-LDL-cholesterolemia.

\begin{tabular}{|c|c|c|c|c|}
\hline & Dominant model & Recessive model & Additive 1 model & Additive 2 model \\
\hline \multicolumn{5}{|l|}{ Dyslipidemia } \\
\hline P-value & 0.00024 & 0.0280 & 0.0011 & 0.0177 \\
\hline OR $(95 \% \mathrm{CI})$ & $1.29(1.13-1.48)$ & $1.74(1.07-2.87)$ & $1.26(1.10-1.45)$ & $1.82(1.11-3.00)$ \\
\hline \multicolumn{5}{|c|}{ Hypertriglycedemia } \\
\hline P-value & 0.0035 & 0.7683 & 0.0022 & 0.8968 \\
\hline OR $(95 \% \mathrm{CI})$ & $1.28(1.08-1.51)$ & $0.91(0.49-1.67)$ & $1.30(1.10-1.54)$ & $0.96(0.48-1.76)$ \\
\hline \multicolumn{5}{|c|}{ Hypo-HDL-cholesterolemia } \\
\hline P-value & 0.5678 & 0.4723 & 0.4617 & 0.4920 \\
\hline OR $(95 \% \mathrm{CI})$ & $1.09(0.81-1.44)$ & $0.59(0.10-1.94)$ & $1.12(0.83-1.49)$ & $0.61(0.10-1.98)$ \\
\hline \multicolumn{5}{|c|}{ Hyper-LDL-cholesterolemia } \\
\hline P-value & 0.0254 & 0.1910 & 0.0462 & 0.1553 \\
\hline OR $(95 \% \mathrm{CI})$ & $1.17(1.02-1.35)$ & $1.38(0.84-2.21)$ & $1.16(1.00-1.33)$ & $1.42(0.87-2.28)$ \\
\hline
\end{tabular}

BMI, prevalence of smoking, presence of hypertension and diabetes mellitus, serum concentrations of total cholesterol, triglycerides, LDL-cholesterol and creatinine, fasting plasma glucose levels and blood glycosylated hemoglobin content were higher, whereas the serum concentration of HDL-cholesterol was lower, in the individuals with dyslipidemia compared with the controls. Among the dyslipidemic individuals, 760 subjects (26.1\%) had taken antidyslipidemic drugs.

Genotype distributions and allele frequencies. A comparison of genotype distributions and allele frequencies between individuals with dyslipidemia and the controls using the $\chi^{2}$ test revealed that rs6929846 of BTN2A1 was significantly $(\mathrm{P}<0.05)$ associated with the prevalence of dyslipidemia (Table II). This polymorphism was also significantly correlated with the prevalence of hypertriglycedemia and hyper-LDL-cholesterolemia, but not to hypo-HDL-cholesterolemia (Table II). The genotype distributions of rs6929846 were in Hardy-Weinberg equilibrium among the subjects with dyslipidemia, hypertriglyceridemia, hypo-HDL-cholesterolemia, hyper-LDL-cholesterolemia and all the corresponding controls (Table II).

A multivariable logistic regression analysis adjusted for age, gender, BMI, smoking status and the prevalence of diabetes mellitus revealed that rs6929846 of BTN2Al was significantly 
Table IV. Correlation of rs6929846 of BTN2Al with serum lipid profiles.

\begin{tabular}{|c|c|c|c|c|c|}
\hline \multirow[b]{3}{*}{ Profile } & \multirow{2}{*}{\multicolumn{3}{|c|}{ BTN2A1 genotype }} & \multicolumn{2}{|c|}{ P-value } \\
\hline & & & & \multirow{2}{*}{$\begin{array}{c}\text { Dominant } \\
(\mathrm{CT}+\mathrm{TT} \text { vs. CC })\end{array}$} & \multirow{2}{*}{$\begin{array}{c}\text { Recessive } \\
(\mathrm{TT} \text { vs. } \mathrm{CC}+\mathrm{CT})\end{array}$} \\
\hline & $\mathrm{CC}$ & $\mathrm{CT}$ & TT & & \\
\hline \multicolumn{6}{|l|}{ All individuals } \\
\hline Number of subjects & 4744 & 1139 & 75 & & \\
\hline Serum total cholesterol, $\mathrm{mmol} / \mathrm{l}$ & $5.15 \pm 0.85$ & $5.26 \pm 0.85$ & $5.38 \pm 0.98$ & 0.0007 & 0.0724 \\
\hline Serum triglycerides, mmol/l & $1.25 \pm 0.87$ & $1.32 \pm 0.88$ & $1.23 \pm 0.54$ & 0.0244 & 0.7456 \\
\hline Serum HDL-cholesterol, mmol/l & $1.66 \pm 0.44$ & $1.63 \pm 0.46$ & $1.70 \pm 0.45$ & 0.1604 & 0.2924 \\
\hline Serum LDL-cholesterol, mmol/1 & $3.21 \pm 0.82$ & $3.28 \pm 0.81$ & $3.49 \pm 0.86$ & 0.0017 & 0.0054 \\
\hline \multicolumn{6}{|l|}{$\begin{array}{l}\text { Individuals not taking } \\
\text { antidyslipidemic drugs }\end{array}$} \\
\hline Number of subjects & 4164 & 976 & 58 & & \\
\hline Serum total cholesterol, $\mathrm{mmol} / \mathrm{l}$ & $5.16 \pm 0.85$ & $5.26 \pm 0.86$ & $5.50 \pm 1.03$ & 0.0012 & 0.0131 \\
\hline Serum triglycerides, mmol/l & $1.22 \pm 0.87$ & $1.30 \pm 0.89$ & $1.20 \pm 0.52$ & 0.0181 & 0.7376 \\
\hline Serum HDL-cholesterol, mmol/l & $1.67 \pm 0.44$ & $1.64 \pm 0.46$ & $1.73 \pm 0.49$ & 0.2458 & 0.2676 \\
\hline Serum LDL-cholesterol, mmol/1 & $3.22 \pm 0.82$ & $3.31 \pm 0.81$ & $3.54 \pm 0.88$ & 0.0009 & 0.0055 \\
\hline
\end{tabular}

Bold denotes values $\mathrm{P}<0.05$. BTN2A1, butyrophilin, subfamily 2, member A1 gene; HDL, high-density lipoprotein; LDL, low-density lipoprotein.

associated with dyslipidemia (dominant, reccesive and additive 1 and 2 models), hypertriglycedemia (dominant and additive 1 models) and hyper-LDL-cholesterolemia (dominant and additive 1 models), with the minor $\mathrm{T}$ allele representing a risk for these conditions (Table III). The BTN2A1 genotype was not correlated with hypo-HDL-cholesterolemia.

Among all the individuals, the BTN2A1 genotype was significantly associated with the serum concentrations of total cholesterol (dominant model), triglycerides (dominant model) and LDL-cholesterol (dominant and recessive models) (Table IV). Among the 5,198 individuals who were not taking antidyslipidemic drugs, the BTN2A1 genotype was also significantly associated with the serum concentrations of total cholesterol (dominant and recessive models), triglycerides (dominant model) and LDL-cholesterol (dominant and recessive models).

\section{Discussion}

Previous research over the past four decades has consistently demonstrated the burden of dyslipidemia to be extremely high in terms of morbidity, mortality and medical costs (11), and the World Health Organization estimates that dyslipidemia is associated with more than half of the global cases of coronary heart disease and more than four million mortalities per year (12). Considering that dyslipidemia is a major risk factor of atherosclerotic cardiovascular disease $(3,4,12)$, the prediction of the risk for dyslipidemia on the basis of genetic variants would be beneficial for the personalized prevention of this condition.

In the present study it has been revealed that the $\mathrm{C} \rightarrow \mathrm{T}$ polymorphism (rs6929846) of BTN2Al was significantly associated with dyslipidemia in Japanese individuals, with the $\mathrm{T}$ allele representing a risk factor for this condition. The results demonstrated that individuals with the TT genotype or those with the CT genotype had a 1.82-fold or 1.26-fold increased risk for dyslipidemia, respectively, compared with those with the CC genotype. In addition, individuals with the $\mathrm{T}$ allele had a 1.28 -fold or 1.17-fold increased risk of hypertriglycedemia or hyper-LDL-cholesterolemia, respectively, compared with those with the CC genotype. The BTN2Al genotype was associated with the serum concentrations of total cholesterol, triglycerides and LDL-cholesterol for all individuals, including those who did not take antidyslipidemic drugs, with the $\mathrm{T}$ allele being correlated with increased serum concentrations.

It was previously revealed that the T allele of rs6929846 of BTN2Al was associated with an increased serum concentration of triglycerides and a decreased concentration of HDL-cholesterol in individuals who had visited the studied hospitals (13). Although this polymorphism was associated with the serum concentrations of triglycerides in a previous study (13) and the present study, there were discrepancies between the two. In the present study, the $B T N 2 A 1$ genotype was correlated with the serum concentration of LDL-cholesterol, but not with the serum HDL-cholesterol concentration. This may be attributable to the variation in study populations between the previous (hospital-visiting individuals) (13) and present (community-dwelling individuals) study. The age (65.1 \pm 11.4 and $68.8 \pm 8.9$ years in panels $A$ and $B$, respectively) and the prevalence of diabetes mellitus (31.3 and $38.0 \%$ in panels $\mathrm{A}$ and $\mathrm{B}$, respectively) and hypertension (61.5 and $67.3 \%$ in panels A and B, respectively) were increased among the subjects in the previous study (13) compared with those in the present study.

The BTN2A1 gene is a member of the BTN2 subfamily, which encode proteins belonging to the butyrophilin protein family. While the butyrophilin family was originally 
identified by its ability to aid the production of milk fat globules (14), numerous butyrophilin and butyrophilin-like families of proteins were demonstrated to regulate immune function, and polymorphisms in the coding sequences were correlated with the predisposition to inflammatory diseases (15). Our previous study (8) revealed that the T allele of rs6929846 increased the transcription activity of BTN2A1, and the serum concentrations of high-sensitivity C-reactive protein were significantly greater in individuals in the combined group of CT and TT genotypes than in those with the CC genotype in healthy individuals without neoplastic, infectious or inflammatory disease (16). These observations indicate that the T allele of rs6929846 of BTN2Al may accelerate inflammatory processes.

Inflammation is a key component of regulating lipid metabolism, and it leads to changes in lipid metabolism aimed at decreasing the toxicity of a variety of harmful agents and tissue repair by redistributing nutrients to cells involved in the host defense $(17,18)$. An inflammatory condition under bacterial or viral infection has been shown to result in an increase in the serum concentration of triglycerides and a decrease in the serum HDL-cholesterol $(19,20)$. Multiple cytokines are likely to affect the metabolism of cholesterol or triglycerides through several pathways, including the increased production and decreased clearance of extremely low density lipoproteins, impaired reverse cholesterol transport and decreased excretion of bile acids (17,21-23). Furthermore, the acceleration of the inflammatory process by the T allele of rs6929846 may result in alterations in lipid metabolism, although the underlying mechanism remains to be elucidated.

There were several limitations to the current study: i) Considering that the study subjects comprised only Japanese individuals, further studies are likely to be required that include other ethnic groups; ii) it is possible that rs6929846 of $B T N 2 A 1$ is in linkage disequilibrium with other polymorphisms in $B T N 2 A l$ or in other nearby genes that are actually responsible for the development of dyslipidemia; iii) the functional relevance of rs6929846 of BTN2Al to the pathogenesis of dyslipidemia remains unclear; and iv) environmental factors, including dietary habits, which affect lipid metabolism were not evaluated in the present study.

In conclusion, the current results indicate that BTN2Al may be a susceptibility gene for dyslipidemia in Japanese individuals. The determination of genotype of this polymorphism may prove to be informative for the assessment of the genetic risk for dyslipidemia. Considering that multiple variants, each having a small effect, are likely to ultimately be found to be responsible for a large fraction of the genetic component of dyslipidemia, further identification of dyslipidemia susceptibility genes is likely to allow a more accurate assessment of the genetic components of this condition.

\section{Acknowledgements}

This study was supported by Research Grants from the Japan Health Foundation and Okasan Kato Culture Promotion Foundation.

\section{References}

1. Khera AV and Rader DJ: Discovery and validation of new molecular targets in treating dyslipidemia: the role of human genetics. Trends Cardiovasc Med 19: 195-201, 2009.

2. Ordovas JM: Nutrigenetics, plasma lipids, and cardiovascular risk. J Am Diet Assoc 106: 1074-1081, 2006.

3. Stamler J, Wentworth D and Neaton JD: Is relationship between serum cholesterol and risk of premature death from coronary heart disease continuous and graded? Findings in 356,222 primary screenees of the Multiple Risk Factor Intervention Trial (MRFIT). JAMA 256: 2823-2828, 1986.

4. Pekkanen J, Linn S, Heiss G, Suchindran CM, Leon A, Rifkind BM and Tyroler HA: Ten-year mortality from cardiovascular disease in relation to cholesterol level among men with and without preexisting cardiovascular disease. N Engl J Med 322: 1700-1707, 1990.

5. Aulchenko YS, Ripatti S, Lindqvist I, et al: Loci influencing lipid levels and coronary heart disease risk in 16 European population cohorts. Nat Genet 41: 47-55, 2009.

6. Kathiresan S, Melander O, Guiducci C, et al: Six new loci associated with blood low-density lipoprotein cholesterol, high-density lipoprotein cholesterol or triglycerides in humans. Nat Genet 40: 189-197, 2008.

7. Kathiresan S, Willer CJ, Peloso GM, et al: Common variants at 30 loci contribute to polygenic dyslipidemia. Nat Genet 41: 56-65, 2009.

8. Yamada Y, Nishida T, Ichihara S, et al: Association of a polymorphism of BTN2A1 with myocardial infarction in East Asian populations. Atherosclerosis 215: 145-152, 2011.

9. Yamada Y, Matsuo H, Warita S, et al: Prediction of genetic risk for dyslipidemia. Genomics 90: 551-558, 2007.

10. Itoh Y, Mizuki N, Shimada T, et al: High-throughput DNA typing of HLA-A, -B, -C, and -DRB1 loci by a PCR-SSOP-Luminex method in the Japanese population. Immunogenetics 57: 717-729, 2005.

11. Smith DG. Epidemiology of dyslipidemia and economic burden on the healthcare system. Am J Manag Care 13 (Suppl 3): S68-S71, 2007.

12. World Health Organization: Quantifying selected major risks to health. In: The World Health Report 2002 - Reducting Risks, Promoting Healthy Life. World Health Organization, Geneva, pp47-97, 2002.

13. Fujimaki T, Kato K, Oguri M, et al: Association of a polymorphism of BTN2A1 with dyslipidemia in East Asian populations. Exp Ther Med 2: 745-749, 2011.

14. Ogg SL, Weldon AK, Dobbie L, Smith AJ and Mather IH: Expression of butyrophilin (Btn1a1) in lactating mammary gland is essential for the regulated secretion of milk-lipid droplets. Proc Natl Acad Sci USA 101: 10084-10089, 2004.

15. Arnett HA, Escobar SS and Viney JL: Regulation of costimulation in the era of butyrophilins. Cytokine 46: 370-375, 2009.

16. Oguri M, Kato K, Yoshida T, et al: Association of a genetic variant of BTN2A1 with metabolic syndrome in East Asian populations. J Med Genet 48: 787-792, 2011.

17. Khovidhunkit W, Kim MS, Memon RA, et al: Effects of infection and inflammation on lipid and lipoprotein metabolism: mechanisms and consequences to the host. J Lipid Res 45: 1169-1196, 2004.

18. Esteve E, Ricart W and Frenándes-Real JM: Dyslipidemia and inflammation: an evolutionary conserved mechanism. Clin Nutr 24: 16-31, 2005.

19. Gallin JI, Kaye D and O'Leary WM: Serum lipids in infection. N Engl J Med 281: 1081-1086, 1969.

20. Sammalkorpi K, Valtonen V, Kerttula Y, Nikkilä E and Taskinen MR: Changes in serum lipoprotein pattern induced by acute infections. Metabolism 37: 859-865, 1988.

21. Chen X, Xun K, Chen L and Wang Y: TNF-alpha, a potent lipid metabolism regulator. Cell Biochem Funct 27: 407-416, 2009.

22. McGillicuddy FC, de la Llera Moya M, Hinkle CC, et al: Inflammation impairs reverse cholesterol transport in vivo. Circulation 119: 1135-1145, 2009.

23. Reiss AB: Effects of inflammation on cholesterol metabolism: impact on systemic lupus erythematosus. Curr Rheumatol Rep 11: 255-260, 2009. 